INPLASY

PROTOCOL

To cite: Liu et al. Differential Effects of Vitamin D And Active Vitamin D Supplements for Preventing Falls: A MetaAnalysis. Inplasy protocol 202090018. doi:

10.37766/inplasy2020.9.0018

Received: 04 September 2020

Published: 04 September 2020

Corresponding author:

Zhimin Yang

yangzmting@qq.com

Author Affiliation:

Xiangya School of Medicine Central South University

Support: None.

Review Stage at time of this submission: The review has not yet started.

Conflicts of interest:

The authors declare that they have no competing interests.

\section{Differential Effects of Vitamin D And Active Vitamin D Supplements for Preventing Falls: A Meta-Analysis}

Liu, T1; Yang, Z2; Li, Z3; Ren, X4.

Review question / Objective: Is the Health Qigong effective and safe for anti-aging? Can Health Qigong regulate immune function and enhance the antioxidant capacity? This review is intended to find the answers.

Condition being studied: Aging is a natural process which is generally along with a decline in the physiological function of the body, as a result of the susceptibility to age-associated diseases will be increased.For example, cardiovascular disease, dementia, osteoporosis, diabetes, hypertension, and stroke are age-induced diseases.Aging brings several diseases together will produce destructive effects on human society and breakdowns the entire health care system and economy.

INPLASY registration number: This protocol was registered with the International Platform of Registered Systematic Review and Meta-Analysis Protocols (INPLASY) on 04 September 2020 and was last updated on 04 September 2020 (registration number INPLASY202090018).

\title{
INTRODUCTION
}

Review question / Objective: Is there any difference between Vitamin $d$ and active Vitamin D supplements for preventing falls? What is the better choice for preventing falls?
Rationale: Vitamin D has direct effects on muscle strength. Although it has been identified that vitamin D supplementation could improve strength, function, balance in a dose-related pattern and even reduce falls in several trials for older individuals, 
several trials of vitamin D have had nonsignificant results. Besides, active forms of vitamin D and standard supplemental vitamin $D$ have no direct comparison of in recent years. We aim to detect the effect.

Condition being studied: Each year many people aged 60 years or older experiences at least one fall. Moreover, falls would not only result in serious injury or death, but older adults who experience falls also report increased anxiety and depression and reduced quality of life. Vitamin D has direct effects on muscle strength. Although it has been identified that vitamin D supplementation could improve strength, function, balance in a dose-related pattern and even reduce falls in several trials for older individuals, several trials of vitamin $D$ have had non-significant results. Besides, active forms of vitamin $D$ and standard supplemental vitamin D have no direct comparison of in recent years.

\section{METHODS}

Search strategy: data source: Relevant studies were identified in the following electronic databases: PubMed, Google Scholar, Cochrane Library, Web of Science and Clinical Trials. Clinical trial registries were also searched for unpublished trials. All databases were searched from inception to present. To identify additional trials, we will hand search the bibliographies of all included studies, as well as any reviews on the topic. We will also search conference proceedings related to this topic. Search keywords: randomized controlled trial, vitamin $D$, active vitamin $D$ and falls.

Participant or population: Patients over age 60 years.

Intervention: Vitamin D, with and without calcium.

Comparator: Active vitamin D, with and without calcium.

Study designs to be included: randomized controlled trials.
Eligibility criteria: Randomised controlled trials of fall prevention with a defined oral dose of supplemental vitamin D or oral active vitamin $D$ in individuals aged over 60 years with a minimum follow-up of three months were identified. specific inclusion criteria:(1) double blind trials; (2) the study had to include definition and assessment of falls; (3) trials had to regard falls as primary or secondary end point defined at the onset of the trial and provide all data. Exclusion criteria:(1) control group, observational studies, and animal studies; (2) Patients with a high risk of falling because some diseases, for example, Parkinson's disease, patients with stroke and so on; (3) Intravenous or intramuscular administration of vitamin $D$ or active vitamin D.

Information sources: Relevant studies were identified in the following electronic databases: PubMed, Google Scholar, Cochrane Library, Web of Science and Clinical Trials. Clinical trial registries were also searched for unpublished trials. All databases were searched from inception to present. To identify additional trials, we will hand search the bibliographies of all included studies, as well as any reviews on the topic. We will also search conference proceedings related to this topic.

Main outcome(s): The primary outcomes include the relative risk of having at least one fall among persons older than 60 receiving vitamin $D$ with or without calcium compared with the risk among those individuals receiving active vitamin D with or without calcium.

Quality assessment / Risk of bias analysis: The appraisal was conducted by pairs of independent reviewers, with conflicts resolved by a third reviewer. The quality assessment of RCTs adopts the risk of bias (ROB) assessment tool provided by the Cochrane Handbook. the following methodological features most relevant to the control of bias: randomisation; masking of treatment allocation; blinding; adherence; and withdrawals. 
Strategy of data synthesis: Across all outcomes, pairwise random-effects metaanalysis was conducted. We assessed the differential effects of Vitamin D and active vitamin $D$ supplements for decreasing fall incidence. Review Manager software version 5.5 provided by the Cochrane Collaboration will be performed for data synthesis and analysis. Meta-analysis was performed to calculate risk ratios (RRs), absolute risk differences (ARD) and 95\% Cls using the MantelHaenszel statistical method. Studies reporting dichotomous outcomes with zero events across all groups were included in the systematic review, but excluded from analysis. Studies reporting continuous outcomes with the average effect estimate but not reporting the associated measure of variance were included in the analysis, with standard errors imputed when feasible.

Subgroup analysis: Subgroup analyses undertake for supplemental vitamin D included type of vitamin D (D2v D3), gender, age ( $<80$ years $v \geq 80$ years), treatment duration ( $<12$ months $v \geq 12$ months), level of independence (independent $v$ institutionalised), and additional calcium supplementation. Subgroup analysis was to assess whether the differences between subgroups were statistically significant.

Sensibility analysis: Sensitivity analysis is mainly used to evaluate the tobustness of the primary outcome measures. We identified additional trials that did not meet the primary analysis criteria to be included in sensitivity analysis.

Language: English.

Country(ies) involved: China.

Keywords: Vitamin D; active Vitamin D; Fall.

Contributions of each author:

Author 1 - Tang Liu.

Author 2 - Zhimin Yang.

Author 3 - Zhihong Li.

Author 4 - Xiaolei Ren. 